\title{
Violencia y síntomas depresivos en estudiantes de Psicología
}

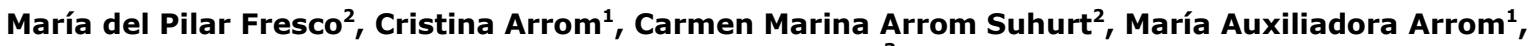 \\ Marcos Capurro ${ }^{3}$
}

${ }^{1}$ Centro para el Desarrollo de la Investigación Científica (CEDIC). Paraguay

${ }^{2}$ Cátedra de Psiquiatría de la Facultad de Ciencias Médicas, Universidad Nacional de Asunción (UNA). Paraguay

${ }^{3}$ Centro Nacional de Control de Adicciones, Ministerio de Salud Pública y Bienestar Social (MSPyBS). Paraguay

Cómo referenciar este artículo/ How to reference this article:
Fresco MP, Arrom C, Arrom Suhurt CM, Arrom MA, Capurro M. Violencia y síntomas depresivos en estudiantes de Psicología. Mem. Inst. Investig. Cienc. Salud. 2018; 16(1): 39-44

\section{R E S U M E N}

El objetivo del estudio fue determinar los antecedentes de violencia intrafamiliar y su relación con depresión en estudiantes de la carrera de psicología. Estudio descriptivo de corte transversal en el que se incluyeron estudiantes del primer al quinto curso de la carrera de Psicología de dos universidades privadas. Previa firma del consentimiento informado se aplicó un cuestionario auto administrado, estructurado y anónimo que incluía datos sociodemográficos, antecedentes de violencia física y sicológica con la escala de Gelles y Strauss y el cuestionario de síntomas de depresión para Atención Primaria de la Salud de la OMS. Los datos fueron cargados en hoja de cálculo de Excel y analizados con el paquete estadístico SPSS. De los 146 estudiantes, el 61,6\% $(n=90)$ tenía entre 18 a 25 años de edad; $82,9 \%(n=121)$ era del sexo femenino; $73,3 \%(n=107)$ solteros/as; $63,6 \%(n=93)$ con actividad laboral; $69,2 \%(n=101)$ profesa la religión católica, $84,2 \% \quad(n=123)$ con actividades recreativas, $58,2 \%(n=85)$ participa en algún grupo u organización social. El $50,7 \%(n=74)$ reportó haber sufrido violencia física y $69,9 \%(n=102)$ violencia psicológica. El $51 \%(n=75)$ presentaba síntomas de depresión. La sintomatología depresiva se asoció con tener antecedente de violencia psicológica $(p=0,006)$ y violencia física $(p<0,008)$. El presente constituye un primer estudio sobre un tema muy actual, poco explorado que tiene la limitación de estudios con muestreo no probabilístico. Por lo cual, requiere ser considerado como una primera aproximación al tema.

Palabras claves: violencia, depresión, estudiante de psicología.

\section{Violence and depressive symptoms in Psychology students}

\section{A B S T R A C T}

The objective was to determine domestic violence experience and its relation with depression in psychology students. Cross-sectional diagnostic descriptive study, which included 1st to 5th year Psychology students from two private universities. After informed consent, a self-administered structure and anonymous questionnaire was applied. The study variables included socio-demographic data, records of physical and psychological violence determined by the scale of Gelles and Strauss and the WHO Primary Care screening questionnaire for depression. Data were loaded in an Excel spreadsheet and then analyzed by SPSS. Out of 146 students, $61.6 \%(n=90)$ was between 18 and 25 years old, $82.9 \%$ $(n=121)$ were female; single 73.3\% $(n=107), 63.6 \%(n=93)$ had a job, 69\% $(n=101)$ was catholic, $84.2 \% \quad(n=123)$ participated in recreational activities, 58.2\% $(n=85)$ have participated in a group or social organization. Seventy four students $(54.7 \%)$ referred having suffered physical violence and $69.9 \%(n=102)$ psychological violence; $51 \%(n=75)$ had depression symptoms. Depressive symptomatology was related with psychological violence $(p<0006)$ and physical violence $(p<0.008)$. This is the first study on a very current 
but little explored issue which has the limitation of studies with non-probabilistic sampling. Therefore, it needs to be considered as a first approach to the theme.

Keywords: violence, depression, psychology students.

\section{INTRODUCCIÓN}

La expresión violencia doméstica es utilizada en numerosos países para hacer referencia a la violencia de pareja íntima, aunque también puede abarcar el maltrato infligido a niños y ancianos 0 al maltrato por cualquier integrante de la familia ${ }^{(1,2)}$. La violencia perpetrada contra la mujer es una pandemia global que afecta hasta al $70 \%$ de la población a nivel mundial y es calificada como una violación de derechos humanos. Para Naciones Unidas la violencia contra la mujer es "todo acto de violencia de género que resulte, o pueda tener como resultado un daño físico, sexual o psicológico para la mujer, inclusive las amenazas de tales actos, la coacción o la privación arbitraria de libertad, tanto si se producen en la vida pública como en la privada"(3-5).

Respecto a la prevalencia a nivel mundial, la OMS estimó que en contextos sin conflictos, mujeres de 15 a 49 años entre $15 \%$ a $71 \%$ refirieron haber sufrido a lo largo de su vida violencia física o sexual de parte de su pareja íntima. En promedio, 30\% de las mujeres que han tenido una relación de pareja han experimentado alguna forma de violencia física o sexual por parte de la pareja íntima y por otro lado, hasta $38 \%$ de asesinatos de mujeres fueron cometidos por sus compañeros ${ }^{(3-5)}$. Entre las consecuencias que tiene para la salud de las mujeres, se pueden describir problemas de salud física, mental, siendo la depresión uno de los trastornos asociados ${ }^{(3)}$.

La depresión es un trastorno mental frecuente, que afecta a 300 millones de personas a nivel mundial y es causa principal de discapacidad, contribuyendo de manera primordial a la carga mundial de morbilidad en países de ingresos altos o bajos ${ }^{(4,5)}$. Las mujeres son más propensas que los hombres a padecer depresión. En China, la tasa de suicidio en mujeres del ámbito rural es mayor que la de varones. Las tentativas de suicidio que generalmente superan a los suicidios consumados (en 20 veces) suelen ser más frecuentes en mujeres y comportan una pesada carga de discapacidad en las mujeres que está pasando inadvertida, representan un factor clave de riesgo de muerte y deben ser atendidas con respuestas de los servicios de salud $^{(4,5)}$.

Un estudio realizado en México en 2014 sobre el perfil de salud mental de estudiantes de psicología encontró un $11 \%$ de patologías mentales que coincidieron con síntomas de ansiedad, depresión y descontrol de impulsos ${ }^{(6)}$. Otro, que se propuso evaluar el riesgo suicida y los síntomas asociados en estudiantes de Psicología, puso en evidencia que 3,6\% tenía alto riesgo de suicidio y 9,1 se había autolesionado alguna vez. Respecto al alto riesgo de suicidio, el hallazgo fue que el de mujeres era tres veces mayor que el de los hombres del estudio ${ }^{(7)}$. Otra investigación realizada en México constató que la violencia intrafamiliar se encontraba asociada con depresión mayor ${ }^{(8,9)}$. Otros estudios realizados en Paraguay ya habían encontrado vinculación entre la presencia de depresión y antecedentes de violencia intrafamiliar o de pareja en población de estudiantes de nivel medio, en mujeres embarazadas y mujeres puérperas; todos asociados al haber padecido violencia intrafamiliar ${ }^{(10-12)}$. Un reciente estudio realizado en 69 estudiantes de psicología de una población universitaria de Asunción encontró prevalencia de $22 \%$ de depresión ${ }^{(13)}$.

\section{METODOLOGÍA}

Se realizó un estudio descriptivo diagnóstico de corte transverso y de carácter cuantitativo $^{(14)}$. La población estuvo compuesta por estudiantes universitarios/as del 1ro. al 5to. curso de la Carrera de Psicología de dos instituciones educativas del sector privado que se encontraban en su institución el día de la encuesta. Se aplicó un cuestionario auto administrado, estructurado y anónimo, con consentimiento informado. Las variables de estudio incluyeron datos sociodemográficos, antecedentes de violencia física y sicológica con la escala de Gelles y Strauss. Para violencia psicológica o emocional, se utilizaron los ítems c, d, e f y g (desde el grito hasta las amenazas) y los ítems h, i, j, k, l, m, n (desde empujar y zarandear hasta usar un arma para agredir) correspondientes a violencia física. Se utilizó el cuestionario de síntomas de depresión para Atención Primaria de la Salud (OMS) para clasificar la sintomatología referida al síndrome depresivo.

Los datos fueron cargados en una hoja de cálculo Excel y posteriormente analizados con el paquete estadístico SPSS versión 15.0 para Windows 7, e incluyó estadística descriptiva y 
analítica. Los resultados se expresaron en forma de frecuencia absoluta y relativa; para indagar posibles asociaciones entre tipos de violencia con características sociodemográficas y depresión se utilizó la prueba de chi cuadrado a un nivel de significancia de 0,05. También se obtuvo el alfa de Crombach para establecer la coherencia interna de los datos en la población de estudio.

Aspectos éticos: antes de ingresar al estudio, los y las estudiantes recibieron información detallada acerca del proyecto y de la disponibilidad de un Servicio gratuito de atención en salud mental, al que podian acudir ante cualquier malestar de tipo emociónal o sintomatología que padecieran durante la aplicación del cuestionario o luego de su participación. Posteriormente firmaron el consentimiento informado con datos de número telefónico y persona de referencia de la investigación, para recurrir en caso de necesitarlo.

\section{RESULTADOS}

La mayoría de la población estudiada, $61,6 \%(n=90)$ tenía entre 18 a 25 años de edad. El $82,9 \%(n=121)$ era de sexo femenino, estado civil soltero $73,3 \%(n=107)$; respecto al año académico, el 30,8\% $(n=45)$ se encontraba en primer curso de la carrera; el $63,6 \%$ $(n=93)$ tenía actividad laboral y de ellos el $70 \%(n=66)$ se desempeñaba como empleado en empresa, $24,7 \%(n=23)$ como trabajador independiente (Tabla 1$)$.

Tabla 1. Características socio-demográficas de los estudiantes de psicología. $n=146$

\begin{tabular}{lll}
\hline $\begin{array}{l}\text { Características socio-demográficas } \\
\text { Edad (años) }\end{array}$ & $\mathrm{n}$ & $\%$ \\
\hline 18 a 25 & 90 & 61,6 \\
26 a 35 & 40 & 27,4 \\
>35 & 16 & 10,9 \\
Sexo & 121 & 82,9 \\
$\quad$ Femenino & 25 & 17,1 \\
Masculino & & \\
Estado civil & 107 & 73,3 \\
Soltero & 27 & 18,5 \\
Casado & 7 & 4,8 \\
Conviviendo & 5 & 3,4 \\
Separado o divorciado & & \\
Año académico & 45 & 30,8 \\
$\quad$ Primer curso & 29 & 19,9 \\
Segundo curso & 24 & 16,4 \\
Tercer curso & 27 & 18,5 \\
Cuarto curso & 15 & 10,3 \\
Quinto curso & 5 & 3,4 \\
Sexto curso & 1 & 0,7 \\
$\quad$ No responde & & \\
Actividad laboral & 48 & 32,8 \\
$\quad$ No tiene & 93 & 63,6 \\
Si tiene & 5 & 3,4 \\
$\quad$ No responde & & \\
Categoría de la ocupación (n=93) & 66 & 70,9 \\
Empleado & 23 & 24,7 \\
Independiente & 2 & 2,2 \\
Funcionario público & 1 & 1,1 \\
Obrero & 1 & 1,1 \\
No responde & & \\
\hline
\end{tabular}

La violencia intrafamiliar, aquella referida en la escala de Gelles que fue clasificada entre los ítems de violencia de tipo psicológico, se presentó en el $69,9 \% \quad(n=102)$ de los encuestados que manifestó haberla padecido por parte de padres/tutores/as o encargados de su crianza y un $50,7 \%(n=74)$ reportó haber sufrido violencia física (Tabla 2$)$. 
Tabla 2. Antecedentes de violencia doméstica en estudiantes de psicología. $n=146$

\begin{tabular}{lll}
\hline Presencia de Violencia & Frecuencia & Porcentaje \\
\hline Violencia Psicológica & 102 & 69,9 \\
Violencia Física & 74 & 50,7 \\
\hline
\end{tabular}

El $51 \%(n=75)$ de los estudiantes tenia síntomas de depresión. Del total $(75 / 146)$ de personas con depresión, 32\% $(n=24)$ tenía sintomatología de episodio leve, 33,3\% $(n=25)$ síntomas de depresión moderada y 34,6\% $(n=26)$ depresión grave. (Tabla 3).

Tabla 3. Grado de depresión en estudiantes de psicología de dos universidades privadas

\begin{tabular}{lll}
\hline Grado de depresión & Frecuencia & Porcentaje \\
\hline Depresión Leve & 24 & 32 \\
Depresión Moderada & 25 & 33,3 \\
Depresión Grave & 26 & 34,6 \\
Total & 75 & 100.0 \\
\hline
\end{tabular}

Para establecer si la presencia de antecedentes de violencia intrafamiliar, específicamente la psicológica, se encontraba asociada a la presencia de sintomatología referida al síndrome depresivo, se utilizó la prueba de chi cuadrado a un nivel de significancia de 0,05. Se encontró correlación entre antecedentes de violencia psicológica y sintomatología referida a depresión $(p<0,06)$. Del mismo modo, para establecer si la presencia de violencia física se encontraba asociada a la presencia de sintomatología referida al síndrome depresivo, se utilizó la prueba de chi cuadrado a un nivel de significancia de 0,05 y se encontró una correlación entre antecedentes de violencia física y sintomatología referida a depresión $(p<0,08)$ (Tabla 4).

Tabla 4. Antecedentes de Violencia Psicológica y Depresión y Antecedentes de Violencia Física y Depresión. $\mathrm{n}=146$

\begin{tabular}{llcr}
\hline \multicolumn{2}{c}{ Antecedentes de violencia } & Con depresión & Valor p \\
\hline Violencia psicológica & $\operatorname{Con}(n=102)$ & $60(58,8 \%)$ & 0,06 \\
& $\operatorname{Sin}(n=44)$ & $15(34 \%)$ & \\
Violencia física & $\operatorname{Con}(n=74)$ & $46(62 \%)$ & 0,08 \\
& $\operatorname{Sin}(n=72)$ & $29(40,3 \%)$ & \\
\hline
\end{tabular}

El alfa de Crombach que midió la fiabilidad de la escala de medición y coherencia interna de las respuestas de la población de estudio fue de 0,8 lo que corrobora que las respuestas brindadas en las escalas de Gelles y el listado de ítems de sintomatología depresiva de Atención Primaria de la Salud tuvieron esta coherencia y son instrumentos suficientemente confiables.

\section{DISCUSIÓN}

Más de la mitad de la población de estudio fue víctima de violencia física en el proceso de crianza por parte de sus padres o encargados y tres tercios aproximadamente, víctima de violencia psicológica. Estos datos coinciden con estudios como el de multipaís realizado en una población de 24.000 mujeres de 10 países donde se encontró una prevalencia entre 13 a $61 \%$ de violencia física y entre 20 a $75 \%$ de maltrato emocional ${ }^{(2)}$. También coinciden con la encuesta de demografía y salud del CEPEP (2008) que mostró una prevalencia de violencia física de $17,9 \%$ y verbal de $36,0 \%{ }^{(15)}$. Otro realizado a nivel nacional de UNICEF $2012^{(16)}$ refirió que el $61 \%$ de niños y niñas paraguayos fue víctima de algún tipo de violencia intrafamiliar. El $35 \%$ de esos casos constituían violencia física grave. El estudio realizado con niños, niñas y adolescentes entre 2010 y 2012 en el Servicio de Psiquiatría de la Facultad de Ciencias Médicas encontró una prevalencia de violencia psicológica de 69,7\% y violencia física de $64 \%{ }^{(17)}$. En un estudio realizado en 254 pacientes adultos en psicoterapia en un servicio público de psiquiatría se encontró una prevalencia de pacientes con trastornos del estado de ánimo, y una prevalencia de violencia psicológica de $40 \%$ y 
física de $29,7 \%^{(18)}$. En otro estudio llevado adelante en el 2010 con 100 mujeres embarazadas que consultaron en un hospital público, 38\% tenía antecedentes de haber sido víctima de castigos físicos de parte de su madre o encargados de su crianza ${ }^{(19)}$.

Tres cuartas partes de la población de estudio tenían sintomatología referida a síndrome depresivo y se distribuyeron en partes iguales entre episodios depresivos leve, moderado y grave. La sintomatología de síndrome depresivo encontrada fue elevada en comparación con otros estudios en población de estudiantes de psicología a nivel internacional ${ }^{(6-8)}$. Los porcentajes de prevalencia más elevados se encuentran generalmente en poblaciones de servicios de atención pública como hospitales y centros especializados como la investigación realizada en Paraguay entre marzo y agosto del 2014 en un hospital público, que encontró una prevalencia de $69 \%$ de distintos grados de depresión ${ }^{(20)}$.

La vinculación entre el padecimiento de violencia física y psicológica a la presencia de sintomatología referida a depresión se constató en el presente estudio y constituye un elemento clave de hallazgos de estudios que expresan las graves consecuencias que tiene para numerosas poblaciones principalmente de mujeres, la exposición a violencia intrafamiliar durante distintas etapas de su desarrollo. Así lo muestran distintos estudios internacionales ${ }^{(9)}$ y otros a nivel nacional ${ }^{(10-13)}$.

El presente constituye un primer estudio sobre un tema muy actual, poco explorado que tiene la limitación de estudios con muestreo no probabilístico, que, al igual que muchas otras investigaciones que han constituido esfuerzos para analizar la violencia y su vinculación con trastornos de salud mental, no nos permiten llegar a conclusiones significativas respecto a la problemática a nivel nacional.

Estos resultados nos señalan la necesidad de detectar o identificar la presencia de trastornos depresivos en los primeros años de carrera en poblaciones de estudiantes que se asocien a prácticas de violencia en su círculo familiar, los que, sumados a otros factores, estarían provocando padecimientos que requieren pronta intervención. En el caso de las mujeres, como población mayoritariamente afectada por violencia, podría evitarse que se encuentre aislada e incapacitada para realizar actividades laborales cotidianas, arriesgar estudios y actividad laboral. Contribuiría a mejorar el autocuidado y del entorno inmediato ${ }^{(21)}$.

Otro aspecto que nos señalan estos hallazgos tiene relación con la necesidad de que la temática de violencia y principalmente la violencia basada en género, pueda ser incluida en las carreras de Ciencias Sociales, Humanidades, Ciencias Médicas y todas las vinculadas a áreas sociales y de la salud.

El estudio requiere ser considerado como una aproximación al tema de violencia y depresión en futuros profesionales de salud mental. Es necesario implementar mayores investigaciones para comprender los trastornos que se presentan en estudiantes universitarios/as principalmente en carreras de salud mental, que establezcan la frecuencia encontrada, así como los factores asociados.

\section{REFERENCIAS BIBLIOGRÁFICAS}

1. Arrom C. Violencia doméstica: una pandemia resistente. Editorial, Mem Inst Investig Cienc Salud 2015;13(3):4-5.

2. Organización Mundial de la Salud-Oficina Regional para las Américas-Organización Panamericana de la Salud [Internet]. Washington D.C., EEUU: OPS-OMS; [citado el 2 abril de 2014]. Disponible en: http://apps.who.int/iris/bitstream/10665/98816 /1/WHO_RHR_12.36_spa.pdf?ua = 1

3. Organización Mundial de la Salud [Internet]. Ginebra-Suiza: OMS; [citado 12 de agosto de 2013]. Disponible en: http://www.who.int/mediacentre/factsheets/fs2 39/es/

4. OMS. Salud de la mujer. Organización Mundial de la Salud, Washington DC. 2013. Nota descriptiva $\mathrm{N}^{\circ} 334$. Disponible en: http://www.who.int/mediacentre/factsheets/fs3 34/es/
5. OPS/OMS. Pinta el mundo de naranja: pon fin a la violencia contra mujeres y niñas. Secretario General de las Naciones Unidas, Ban Ki-moon. 2017. Disponible en: http://www.paho.org/clap/index.php?option =co m_content\&view $=$ article\&id =343: pinta-elmundo-de-naranja-pon-fin-a-la-violenciacontra-mujeres-y-ninas\&Itemid $=215$ \&lang $=e s$ 6. González Trujillo K, Gaspar Candia P, Luengo López S, Amigo Vázquez Y. Salud mental en estudiantes de la carrera de psicología. Implicancias para la formación en autocuidado de los psicólogos en formación. Integración Académica en Psicología 2014; 2(6). ISSN: 2007-5588.

7. González Sepúlveda PC, Medina Pérez ÓA, Ortiz Valencia JC. Riesgo suicida y factores asociados en estudiantes de Psicología en una Universidad pública de Colombia. Rev haban cienc méd [Internet]. 2016 Feb 
[citado 2017 Jun 22]; 15(1): Disponible en: http://scielo.sld.cu/scielo.php?script=sci_arttex t\&pid=S1729-519X2016000100015\&Ing=es.

8. Bartra Alegría AF, Guerra Sánchez EF, Carranza Esteban Rr. Autoconcepto y depresión en estudiantes universitarios de una universidad privada. (Spanish). Apuntes Universitarios: Revista De Investigación 2016; 6(2), 53-68.

9. Castillo-Manzano RM, Arankowsky-Sandoval G. Violencia intrafamiliar como factor de riesgo para trastorno depresivo mayor en mujeres: Estudio de casos y controles. Revista Biomedica 2008; 19(3), 128-36.

10. Arrom C, Samudio M, Ruoti M, Orúe E. Síndrome depresivo en la adolescencia asociado a género, abuso sexual, violencia física y violencia. Mem Inst Investig Cienc Salud 2015;13(3): 39-44.

11. Arrom C, Ruoti $M$, Samudio $M$, Orué $E$, Arrom CM. Sintomatología depresiva en embarazadas víctimas de abuso sexual. Mem Inst Investig Cienc Salud 2015;13(3):82-7.

12. Arrom C, Samudio M, Arrom CM, Lampert $\mathrm{N}$, Arrom MA, Orué E. Violencia intrafamiliar, de pareja íntima y abuso sexual en puerperio inmediato. Su relación con depresión. Revista Paraguaya de Psiquiatría 2013; 1(2): 17-23.

13. Ruíz Díaz H, Insfrán K, Andrada F, Ayala J. Investigación sobre la prevalencia de Ansiedad y Depresión en estudiantes de psicología y su relación con los rasgos de Personalidad. Revista Científica de la UCSA 4(1), 2017:17-28.

14. Hernández Sampieri R y Otros. Metodología de la Investigación. 5a. Ed. Peru: Mc Graw Hill/Interamericana Editores, S.A. De C.V. Empresa Editora El Comercio S.A. 2010:118-49. 15. Centro Paraguayo de Estudios de Población CEPEP. Encuesta nacional de demografía y salud sexual y reproductiva 2008, ENDSSR 2008. Informe final. Octubre de 2009. Asunción, Paraguay. Disponible en: http://pdf.usaid.gov/pdf_docs/Pnadr811.pdf

16. UNICEF. Asunción-Paraguay: Base Educativa y Comunitaria de Apoyo (BECA), UNICEF; [Internet] [citado 11 de junio 2013]. Disponible en PDF: http://www.unicef.org/paraguay/spanish/py_re sources_Estudio_Maltrato.pdf

17. Arrom C, Arce A, Arrom CM, Fresco MP, Samudio $M$, Capurro $M$ et al. Violencia intrafamiliar en pacientes en edad pediátrica que recibe atención psicológica. Frecuencia, factores predisponentes y consecuencias. Mem. Inst. Investig. Cienc. Salud. 2015;13(3):24-30. 18. Fresco MP, Arrom C, Samudio M, Arrom CM, Capurro M, Arrom MA, et al. Psicopatología en adultos con antecedentes de violencia intrafamiliar. Revista Paraguaya de Psiquiatría. 2015; 3(1):8-12.

19. Galeano $A$, Orrego $B$, Pérez $M$, Ruiz $V$, Arrom MA, Ruoti $M$, et al. Violencia intrafamiliar sufrida por mujeres en un Hospital Público. Revista Paraguaya de Psiquiatría. 2015; 3(1):42-9.

20. Ortiz Cuquejo LM, Samudio Domínguez GC, Avalos DS, González AM. Prevalencia de síntomas depresivos en pacientes pediátricos internados en un Hospital de tercer nivel. Pediatr. (Asunción), 2016; 43(2): 109-14.

21. Organización Mundial de la Salud-Escuela de Higiene y Medicina Tropical de Londres. [Internet]. Washington D.C.: OPS-OMS; [citado 10 Mayo 2014]. Disponible en: http://apps.who.int/iris/bitstream/10665/44810 /1/9789275316351_spa.pdf 